\title{
Automated block-by-block correction of milling parameters by means of numerical simulation
}

\author{
Igor Kiselev ${ }^{1, *}$, Nikita Jukov ${ }^{1}$, and Konstantin Deev ${ }^{2}$ \\ ${ }^{1}$ Bauman Moscow State Technical University, Applied Mechanics department, 105005, Moscow, \\ Russian Federation \\ ${ }^{2}$ Central Aerohydrodynamic Institute named after N.E. Zhukovsky, 140180, Zhukovsky, Moscow \\ region, Russian Federation
}

\begin{abstract}
The methodology of the block-by-block correction of feed during the multi-axis milling process using the criterion based on the calculation of local maxima of the cut-off thickness, which is calculated instantly along the cutting edges, has been introduced in this paper. This correction is carried out using numerical simulation, which is based on the depth buffer and special geometric algorithm. The example of heavy stock removal cycle optimization during three-axis milling of figurine-shaped surface has been given also. This example demonstrates that peak loads on the tool has decreased, whereas the process efficiency has improved by more than one third.
\end{abstract}

\section{Introduction}

Nowadays, the figurine-shaped parts milling takes place using multi-coordinate CNCcontrolled machines. The usage of such kind of equipment, taking into consideration the modern CAM systems capabilities of automatically predicting the tool trajectory, allows to obtain high quality and accuracy of workpiece machined surface due to minimization of the number of workpiece repositioning while processing. The optimal milling modes are to be chosen to increase the process efficiency. It is usually needed to take into account cutting speed and allowable feed (for the pair «tool-machined surface») directory data to set the right milling mode. In the first stage the directory modes are set along the whole process trajectory length except the sections of fast movements and approach mode/disengagement. In the second stage the tryout of the program is carried out through test workpieces. During this tryout, a work planner and a machine operator are to execute manual adjustment taking notice of the acoustic noise, the level of machine vibration, chips, tool wear etc. Invariable feed modes which are set as a result of the tryout are usually too much conservative, because they guarantee the pass of the most difficult machining zones, in which instantaneous values of cut-off thickness or tool coverage are significant. As for other machining zones, the tool is overstressed. It causes drop in productivity compared to potentially obtainable characteristics in case of adaptive modes correction. The manual correction of the machining modes along the process trajectory is impossible in case of

*Corresponding author: i.a.kiselev@yandex.ru 
complicated 3D geometry of the machined workpiece, because the trajectory may consist of dozens or hundreds of thousands blocks. The potential of modern CAM systems is usually limited by feed correction ways depending on the trajectory curvature (mainly when processing inner corners in the three-axial mode) and also the real geometry of the machining zone and the cut-off parameters are not taken into consideration. That's why the correction results cannot be trusted. Therefore, the development of special numerical methods and software which allows to carry out automatic feed correction along the process trajectory, based on the detailed analysis of the cutting zone geometry, is the issue of high relevance. This task is especially important both during the roughing, when it is needed to remove large amount of material with high loads on the tool, and the semifinish machining, when the removal of stepped stock allowance (after roughing) is carried out.

The issues of the cutting process modeling [1-11] and feed correction during milling have been examined in the papers of foreign and home authors.

The approaches represented in the references can be divided into two groups depending on the feed optimization criterion:

1)The first group [12-14] is dedicated to the instantaneous material removal rate (MRR - Material Removal Rate), which is the volume of removed material per time unit. To minimize the machining time, these first group algorithms tend to increase the feed value in the trajectory domains where MRR is lower than the target level and to decrease the feed value in the domains where MRR is higher than the target level. It leads to keeping the integral load on the tool during machining at the same level.

2)The second group [1,15-17] is dedicated to the criterion based on the value of the cutting force affecting the tool. To minimize the machining time, these second group algorithms tend to increase the feed value in the trajectory domains where cutting forces are lower than the target level and to decrease the feed value in the domains where cutting forces are higher than the target level. It leads to keeping the integral load on the tool during machining at the same level.

This paper proposes another block-by-block feed optimization criterion, which is based on the maximum value of local instantaneous cut-off thickness. This criterion defines the local maximum of the load on the tool cutting edges in the machining trajectory blocks. The universal methodology of numerical modeling has been proposed in this paper to calculate the needed value along the machining trajectory. This methodology was designed by the authors in good time to predict the stability of compliant thin-walled parts during milling. The example of feed optimization during the figurine-shaped part milling with the usage of this criterion has been given in the final part of this paper.

\section{The numerical model of machining process}

The software package 3DCUT [5,6,9], which was designed by the authors of this paper for modelling of the figurine-shaped compliant parts machining dynamics, was proposed to put in use to draw the diagrams of instantaneous values of cut-off thickness along the tool cutting edges. These diagrams are needed to calculate the value of the criterion for the feed optimization. The modelling algorithm 3DCUT has got the modular design which includes the following: the special algorithm of geometric modeling, the finite-elements models of the tool and the machining workpiece dynamics (taking into consideration stock allowance removal), the cutting forces model and the module of the nonlinear motion equations integration (paying attention to time lag). The diagrams of cutting forces, tool and workpiece vibrations, the cut-off parameters along the machining trajectory and also the machined surface having taken into account vibrational indentations and form deviation can be obtained as the results of modeling (depending on the machining modes). 
To fulfill the aims of this research, the module of geometric modeling is enough, since the dynamics issues are not taken into account because of sufficient rigidity of technological system components during roughing and semifinish machining (when the feed correction is especially of high relevance). The geometry modeling algorithm 3DCUT was designed by the authors using multilevel depth buffer as a base. It allows to calculate the positional relationship of the tool and the machining surface, draw the diagrams of instantaneous values of cut-off thickness along the cutting edges of the tool, execute the surface geometry alterations during stock allowance removal. The peculiarity of this algorithm is the capacity of analyzing the geometry of the cut-off layer for every cutting edge of the tool at each moment of time. The other geometry modeling algorithms in CAM systems carry out calculations only for rotary body of the tool.

\section{The methodology of feed optimization}

The main criteria of feed optimization, which are described in the introduction, define the integral load on the tool, whereas the tool efficiency, the edges spalling and their wear rate depends on local loads on the smallest parts of the cutting edges. That is why the two types of optimization errors may arise.

The most common example of the first type errors is the process of the stock allowance smoothing by sphereconical end milling cutters after the three-axial layer-by-layer roughing by cylindrical cutters. In this case sphereconical tool cuts off the corner steps, which have been left after roughing. Meanwhile, the tool coverage in the radial direction and the cutting zone proportions along the generatrix are relatively small, but the instantaneous cut-off thickness in the local domain of the cutting edge may significantly exceed the allowable limits, which leads to more rapid tool wear. This example shows that the small size of the contact mark in the cutting domain leads to the low values of MRR and the resultant cutting force, whereas the values of local instantaneous cut-off thickness will be high. This won't allow already existing algorithms slow down the feed to the needed extent in such trajectory domains and the tool will be locally overloaded.

The second group errors may arise during roughing with a high value of the axial depth or/and the circumferential tool coverage and a low value of the radial milling depth. In this case the value of MRR and the resultant cutting forces will be high, whereas the value of instantaneous cut-off thickness on the elementary segments of the cutting edges will be low. The already existing algorithms don't allow to increase the process efficiency and use the tool capacity to the needed extent. If the tool rigidity or the machining workpiece is not high enough, it is needed to take notice of the dynamic stability of the machining process criteria to avoid dangerous self-excited vibrations. The issue of the dynamic stability is mainly of high relevance during the finishing because more rigid tool and workpiece, which maintains the sufficient rigidity due to large amount of stock material, are put into use during the roughing process.

Taking into account the described disadvantages of the integral criteria, the criterion which can be defined by the formula (1) and related to the maximum local load on the elementary segments of the cutting edges is proposed in this paper.

$$
C_{h}^{k}=\max _{s \in\left[s_{k}-\Delta s_{k, k-1}, s_{k}+\Delta s_{k, k+1}\right]}\left(\max _{\substack{i=1 \ldots N_{e} \\ j=1 \ldots N_{p}}}\left(h_{i j}\right)\right)
$$

where $C_{h}$ - the main value of the criterion; $h_{i j}$ - the instantaneous cut-off thickness at the discretization point $j$ of the cutting edge $i ; N_{e}$ - the number of the tool cutting edges; 
$N_{p}$ - the number of the discretization points along the tool cutting edges; $s$ - the current path along the machining trajectory; $k$ - the number of the machining trajectory block; $\Delta s_{m, n}$ - the half of the machining trajectory length between two blocks $m$ and $n$.

The calculation of the main criterion value (unlike the integral criteria) using the formula (1) is possible when the special geometry algorithm is applied to every cutting edge. This requirement is fulfilled in the program software 3DCUT. The higher modelling accuracy in this case leads to significant increase in the need for computing resources and calculation time in comparison with the conventional integral criteria.

The feed correction algorithm includes the following main stages:

1)The calculation of the criterion $C_{h}^{k}$ at the discretization points of the machining trajectory using the geometry modeling algorithm, section 2 .

2) The feed correction in the machining trajectory blocks using the formula (2)

$$
F_{k}=F_{k}^{0} \frac{C_{h}^{0}}{C_{h}^{k}}, \quad F_{k}>F_{\text {max }}: F_{k}=F_{\text {max }}
$$

where $F_{k}^{0}$ - the value of the feed in the trajectory block $k$ before the correction; $F_{k}$ - the value of the feed after the correction; $C_{h}^{0}$ - the target value of the criterion (this value is set assessing the durability of the tool machining the chosen make of the material); $F_{\max }$ - the maximum allowable feed value which is set assessing the machine capability).

3) The feed recorrection in the trajectory blocks to limit the maximum feed acceleration along the trajectory. The allowable value of the acceleration depends on the machine capability and can be determined experimentally during the trajectory idling. The moderate exceed in the allowable acceleration value doesn't lead to the negative consequences for the machine, because in this case the CNC post automatically limit the acceleration values so that they could be feasible by the machine feed in this stage of the process.

4) The recalculation of the criterion $C_{h}^{k}$ using the numerical modeling takin into account the corrected feed values to check the correction and assess the decrease in the processing time.

5) Postprocessing and refining of the trajectory using the $\mathrm{CNC}$ machine

\section{The feed optimization example during the milling process}

The roughing of the workpiece, the geometry of which is two spherical surfaces coupling with different diameters, is given here as an example of the block-by-block feed optimization (figure 1a). The diameter of the workpiece is $60 \mathrm{~mm}$. The workpiece is made of steel 30ХГСА (in Russian). The figure $1 \mathrm{~b}$ shows the roughing process trajectory using the layer-by-layer method in the three-axial mode. The trajectory is calculated by the CAM system Siemens NX 10.0. In each layer the trajectory is concentric circles with the set radial step. The bull-nose tool (the radius of the body equals to $3 \mathrm{~mm}$, has got 5 cutting edges with 38 degrees lifting angle of helix) is applied for processing here. This kind of tool can be found in the catalog of the Iscar company. The figure 1c shows the machined surface picture and the tool cutting edges, which are obtained in the intermediate stage of the modeling in the software package 3DCUT designed by the authors. 


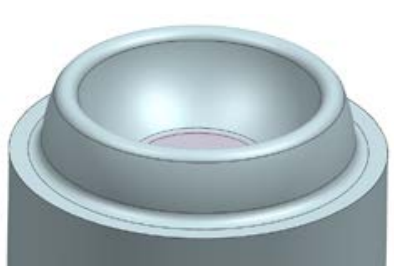

(a)

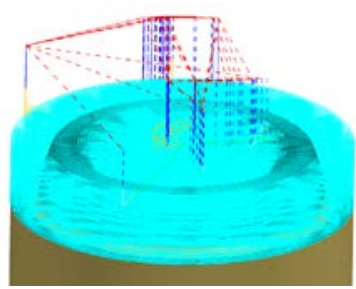

(b)

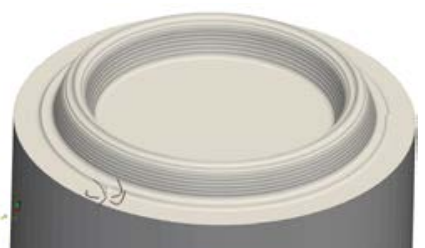

(c)

Fig. 1. The tryout workpiece: $1 \mathrm{a}$ - the target geometry, $1 \mathrm{~b}$ - the machining trajectory, $1 \mathrm{c}-$ modelling in the software package 3DCUT

The figure 2 shows the results of the criterion value $C_{h}$ calculation using the formula (1). These results are represented as dependence on the time of the machining process for the whole trajectory (fig. 2a), for the one layer of trajectory (fig. 2b) and for the one of concentric trajectory curls (fig. 2c). These figures show that moving from one trajectory curl or layer to another one there is a sudden increase in the criterion value $C_{h}$ (the instantaneous cut-off thickness maximum). Such kind of increase may be the indication of the tool overload and that the loads are not high enough during machining. If the permanent mode is chosen, it is needed to limit the feed having taken into account the loads peaks. As it is shown in the fig. $3 \mathrm{a}$, it leads to the overall process efficiency decrease. However, the feed correction algorithm, which is described in part 3 of this article, allows to automatically calculate corrected feed values in the blocks of the trajectory (fig. 3b). The figure $3 \mathrm{c}$ shows the calculated law of the feed change along one of the concentric trajectory curls. In the regular section of the curl the feed value increases due to the allowable machine feed acceleration (in this example this acceleration equals to $2000((\mathrm{~mm} / \mathrm{min}) / \mathrm{s})$ to reach the target value of the maximum cut-off thickness. Then, when the change in curls happens, the sudden geometry change takes place in the cutting zone, which leads to extra load on the tool. The target value of the maximum cut-off thickness $C_{h}^{0}$ equals to 65 $\mu \mathrm{m} /$ tooth, which leads to the minimum tool wear. This value was chosen by the authors and is based on their own experience. The tool rotating frequency equals to $5000 \mathrm{rpm}$. Based on the trajectory with the calculated feed correction, remodeling of the criterion $C_{h}$ has been carried out to assess the machining modes optimization results. The modeling results, which are represented in the fig. 4, shows the significant smoothing of the peaks in the maximum cut-off thickness (fig. 4a) and ending up at the level of the target value $C_{h}^{0}$ along the machining trajectory concentric curls. The machining time has decreased from $2955 \mathrm{~s}$ to 1830 s. It dropped by $38 \%$.

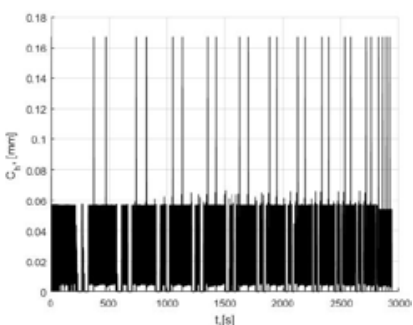

(a)

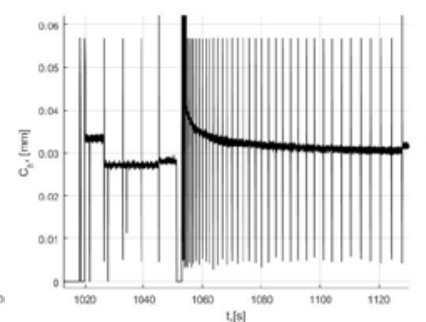

(b)

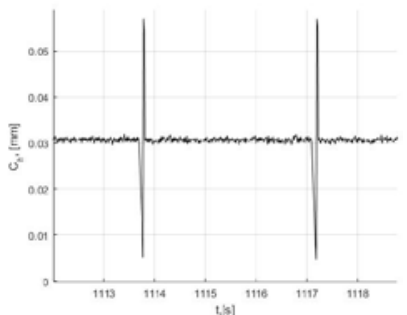

(c)

Fig. 2. The values of maximum local instantaneous cut-off thickness $C_{h}$ for the original machining mode 


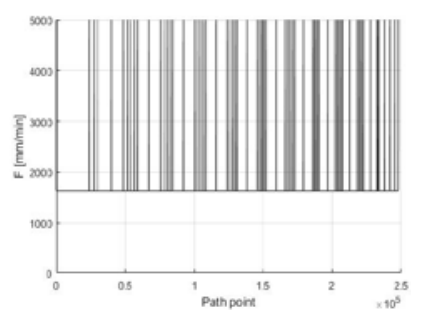

(a)

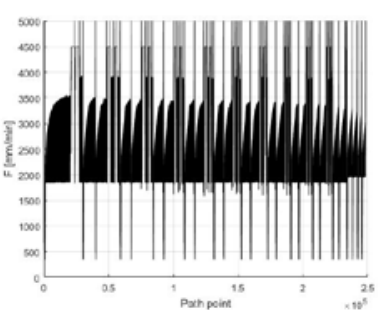

(b)

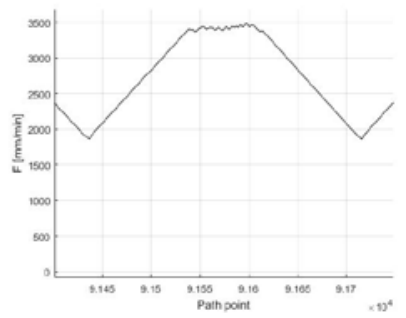

(c)

Fig. 3. The figures of the feed change in dependence on the machining trajectory block number before the correction (a) and after the correction (b, c)

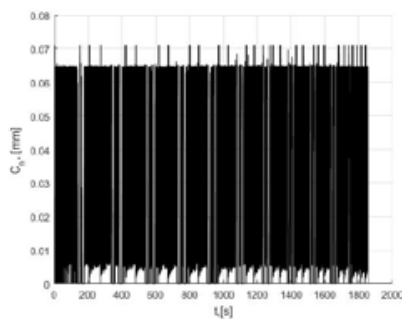

(a)

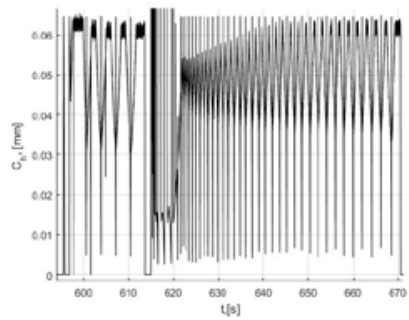

(b)

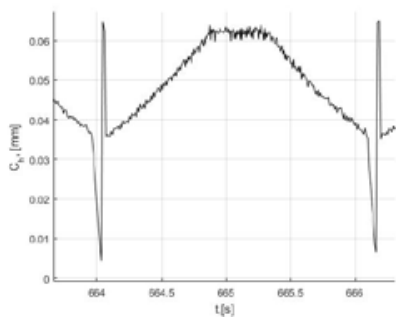

(c)

Fig. 4. The values of local maximum instantaneous cut-off thickness $C_{h}$ after the correction

The corrected modes are automatically outputted as the feed instruction in each block by CL-data files. These files can be then used to postprocess G-code for the concrete machine made by the means of CAM system. It is needed to say that the described feed correction doesn't lead to the significant extra loads on the machine drive units because all of them function in the acceleration / deacceleration mode even in case when the feed doesn't change on the curvilinear trajectory. The acceleration / deacceleration mode doesn't decrease the process efficiency and does lead to the drive units' optimal working conditions.

\section{Conclusion}

The presented in this article approach to the automatic block-by-block feed values calculation during the multiaxial milling allows to significantly increase the process efficiency and at the same time decrease the peak loads on the tool. The proposed criterion of the feed optimization relies on the local values of maximum instantaneous cut-off thickness along the tool cutting edges and in this way allows to execute modelling with higher accuracy compared to the integral criteria. The described example of the machining modes optimization demonstrates the efficiency increase by $38 \%$ and at the same time the peak loads on the tool significantly decrease. The proposed approach might be expanded in the foreseeable future by the speed of rotation limitation taking notice of the dynamic stability of the thin-walled workpieces machining, when the dangerous self-excited vibrations may appear. 


\section{References}

1. R. Jerard et al., Proceedings of the 2000 NSF Design and Manufacturing Research Conference, pp. 3-6 (2000)

2. S. Merdol, Y. Altintas, Journal of Manufacturing Science and Engineering, v. 130(5), p. 051004 (2008)

3. S. Merdol, Y. Altintas, Journal of Manufacturing Science and Engineering, , v. 130(5), p. 051005 (2008).

4. H. Erdim et al. Procedia CIRP,v. 1, pp. 96-101 (2012)

5. F. Anania, A. Pena, M. Zapciu, Tehnicki vjesnik, v. 24(4), pp. 987-992 (2017)

6. I. Kiselev, S. Voronov, Proceedings ASME 2014 International Design Engineering Technical Conferences and Computers and Information in Engineering Conference, p. V006T10A075 (2014)

7. I. Kiselev, S. Voronov, S. Arshinov, Proceedings ASME 2014 International Design Engineering Technical Conferences and Computers and Information in Engineering Conference, p. V006T10A076 (2014)

8. A. Gerasimenko, M. Guskov, A. Gouskov, P. Lorong, A. Shokhin, International Journal of Machining and Machinability of Materials, v. 19(1), pp.17-40 (2017)

9. A. Gouskov et al., International Journal of Machining and Machinability of Materials, v. 19(1), pp. 17-40 (2017)

10. I. Kiselev et al., Procedia Engineering, v. 176, pp. 50-55 (2017)

11. S.Voronov A., M. Veidun, Journal of Machinery Manufacture and Reliability, v. 46(4), pp. 394-403 (2017)

12. L. Qian, B. Yang, S. Lei, Robotics and Computer-Integrated Manufacturing,v. 24(6), pp. 796-803 (2008)

13. K. Erkorkmaz et al. CIRP Annals-Manufacturing Technology, v. 62(1), pp. 395-398 (2013)

14. K. Chan, C. Wang, Automation Science and Engineering, 2015 IEEE International Conference, p. 691-696 (2015)

15. Z. Li, Z. Zhang, L. Zheng, International Journal of Advanced Manufacturing Technology, v. 24(7-8), pp. 541-552 (2004)

16. R. Salami, M. Sadeghi, B. Motakef, International Journal of Machine Tools and Manufacture, v. 47(5), pp. 760-767 (2007)

17. H. Perez et al. Journal of materials processing technology,v. 201(1-3), pp. 486-490 (2008) 